\title{
Homozygous missense mutation in STYXL1 associated with moderate intellectual disability, epilepsy and behavioural complexities
}

Mala Isrie ${ }^{1,2}$, Masoud Zamani Esteki ${ }^{1}$, Hilde Peeters ${ }^{1}$, Thierry Voet ${ }^{1}$, Jeroen Van Houdt ${ }^{1}$, Wim Van Paesschen ${ }^{3}$ and Hilde Van Esch ${ }^{1,2}$

${ }^{1}$ Center for Human Genetics, University Hospitals Leuven, KU Leuven, Leuven, Belgium

${ }^{2}$ Laboratory for the genetics of Cognition, KU Leuven, Leuven, Belgium

${ }^{3}$ Department of Neurology, University Hospitals Leuven, KU Leuven, Leuven, Belgium

Corresponding author:

Hilde Van Esch, MD, PhD

Center for Human genetics

University Hospitals Leuven

Herestraat 49

B3000 Leuven

Belgium

Tel: 0032-16-345903

Fax: 0032-16-346051

Email: Hilde.VanEsch@uzleuven.be

\begin{abstract}
The introduction of massive parallel sequencing has led to the identification of multiple novel genes for intellectual disability (ID) as well as epilepsy. Whereas dominant de novo mutations have been proven to be a leading cause for these disorders, they do not apply to families suggestive of an autosomal recessive inheritance pattern. In this study, we combined the use of linkage analysis with exome sequencing to elucidate the cause of moderate non-syndromic ID, epilepsy and behavioural problems in a consanguineous Iraqi family. A founder missense mutation was identified in STYXL1. We propose this as a novel candidate gene involved in ID, accompanied by seizures and behavioural problems. Our findings further confirm the genetic heterogeneity of cognitive disorders and genetic epilepsy.
\end{abstract}

\section{Keywords}

STYXL1; consanguineous; linkage; exome sequencing 


\section{Introduction}

The introduction of massive parallel sequencing has led to the identification of multiple novel genes for intellectual disability (ID) as well as epilepsy (Barcia et al., 2012, de Ligt et al., 2012, Heron et al., 2012a, Heron et al., 2012b, Rauch et al., 2012). A significant amount of research has focused on the occurrence of dominant de novo mutations, revealed by trio sequencing. Indeed, dominant de novo mutations have been proven to be a leading cause of ID in the Western world (de Ligt et al., 2012, Rauch et al., 2012). However, this approach is not feasible for families with multiple affected individuals in the same generation, which often suggests an autosomal recessive inheritance pattern.

Consanguineous couples with multiple affected children form a distinct group, since linkage analysis in these families often allows to delineate restricted region(s) containing the causal genetic defect. Genetic investigations can therefore focus on a specific region, filtering out many irrelevant variants in the case of whole genome or exome sequencing. An alternative approach is the customized targeting and deep sequencing of the linkage interval only (Najmabadi et al., 2011).

In this study, we combined the use of linkage analysis with exome sequencing to elucidate the cause of moderate non-syndromic ID, epilepsy and behavioural problems in a consanguineous Iraqi family. A founder mutation was identified in a novel candidate gene, further confirming the genetic heterogeneity of cognitive disorders.

\section{Clinical description}

The pedigree of the family is depicted in Figure 1a. The proband (Fig. 1a, V.1) was referred to the department of Paediatric Neurology at the age of 15 years due to behavioural problems and developmental delay accompanied by seizures. Focal epilepsy started at the age of 3 years. Seizures were characterized by vocalisation, upward gaze, clonic movements of the right arm, followed by generalisation. EEGs showed spikes over occipital and frontal derivations and background slowing. MRI of the brain was normal. He was treated with carbamazepine, oxcarbazepine, topiramate, and became seizure free on lamotrigine $250 \mathrm{mg}$ BID and valproate $1 \mathrm{~g}$ BID. He followed special education and at the age of 24 still needed supervision during his general daily activities. Karyotype and FMR1 gene analysis were normal.

Brother V.3 (Fig. 1a) was diagnosed with autism and mild ID and he was admitted to the psychiatric ward at the age of 16 due to psychosis and aggressive behaviour. He was also treated for mild epilepsy since the age of 3 years. He had a few generalized tonic-clonic seizures. EEG showed mild to moderate slow background without epileptic discharges. MRI of the brain was normal. He remained seizure free on valproate $500 \mathrm{mg}$ BID.

Individual V.6 (Fig. 1a) was the youngest affected sibling. He had a delayed speech development, mild ID and epilepsy. The epilepsy started at the age of 5 years. He had focal and generalized tonic-clonic seizures. MRI of the brain was normal. EEG showed frequent multifocal independent spike discharges (MISD) and slow background (Fig. 2). Seizures were controlled on valproate $250 \mathrm{mg}$ TID. He was also diagnosed with an autism 
spectrum disorder (ASD) and showed problematic behaviour including anxiety and sleep disturbance. ArrayCGH was normal.

All three affected siblings were non-dysmorphic. Screening for inborn errors of metabolism showed normal results. Parents were consanguineous and from Asian origin. They also had three unaffected children (V.2, V.4 and V.5, Fig. 1a) and additional family history was negative.

\section{Methods}

Linkage analysis and haplotyping

Genome-wide parametric linkage analysis, as well as haplotyping was performed with Merlin software (Abecasis et al., 2002). A dense SNP marker set derived from the Affymetrix SNP array 6.0 platform was used in a recessive model. As a requirement of Merlin analysis sex-averaged SNP genetic distances derived from deCODE (Kong et al., 2002) were used. Genotyping was done on DNA extracted from peripheral white blood cells, obtained from the parents, their three unaffected and three affected children. Copy number analysis and SNP B-allele frequency computation was carried out by PennCNV and visualised using Circos. (Wang et al., 2007, Krzywinski et al., 2009). 


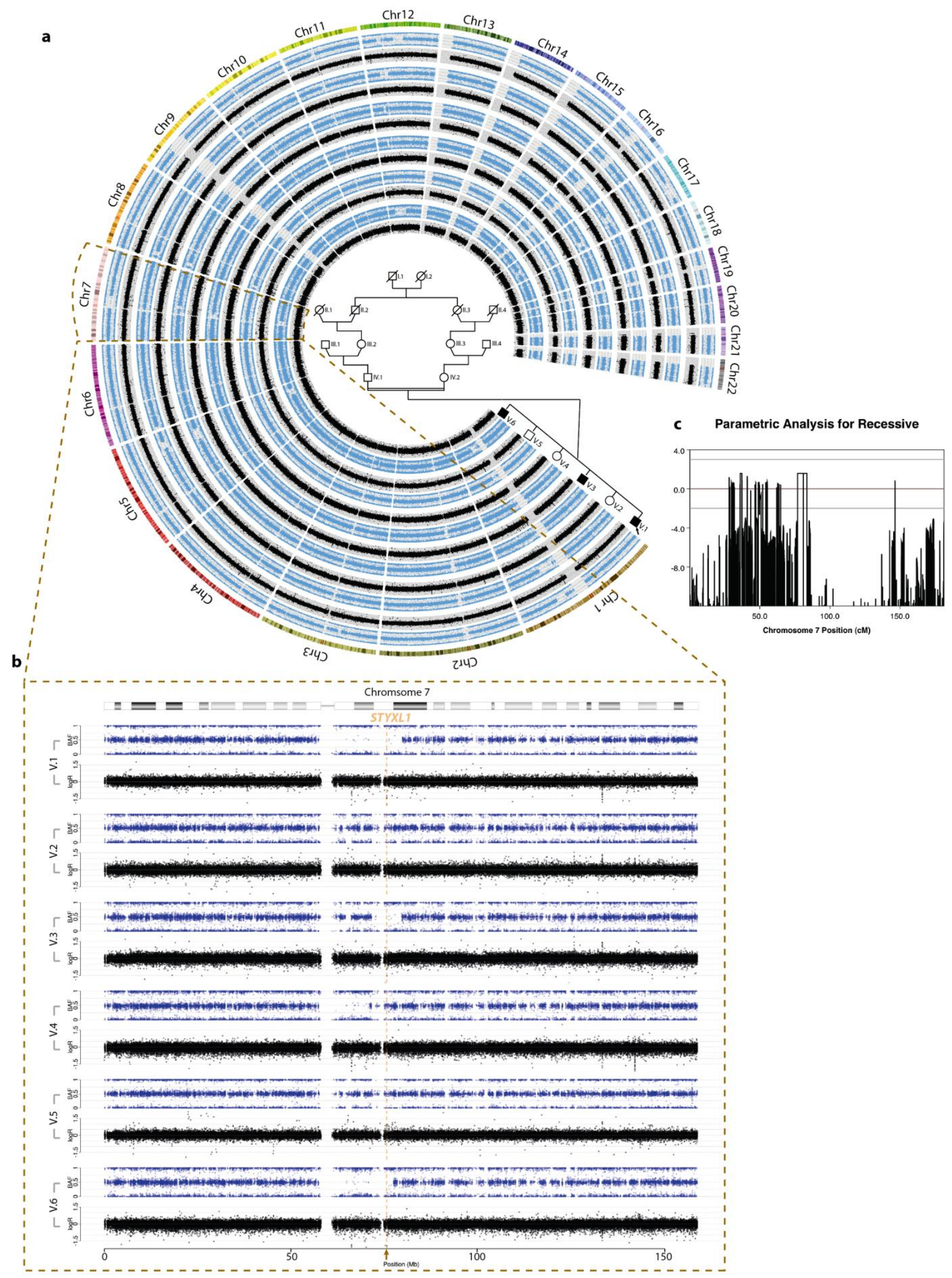

Fig. 1. Pedigree, copy number profiling and linkage-based analysis. a. Genome-wide SNP B-allele fraction and relative copynumber $(\log R)$ profiles of the siblings. The pedigree of the family is depicted in the middle of the Circos-plot. b. A zoomed in illustration of chromosome 7 specific SNP B-allele fraction and relative copy-number (logR) profiles. The physical position of STYXL1 is indicated by an orange arrow and dashed line. c. Linkage-based analysis of Chromosome 7. 

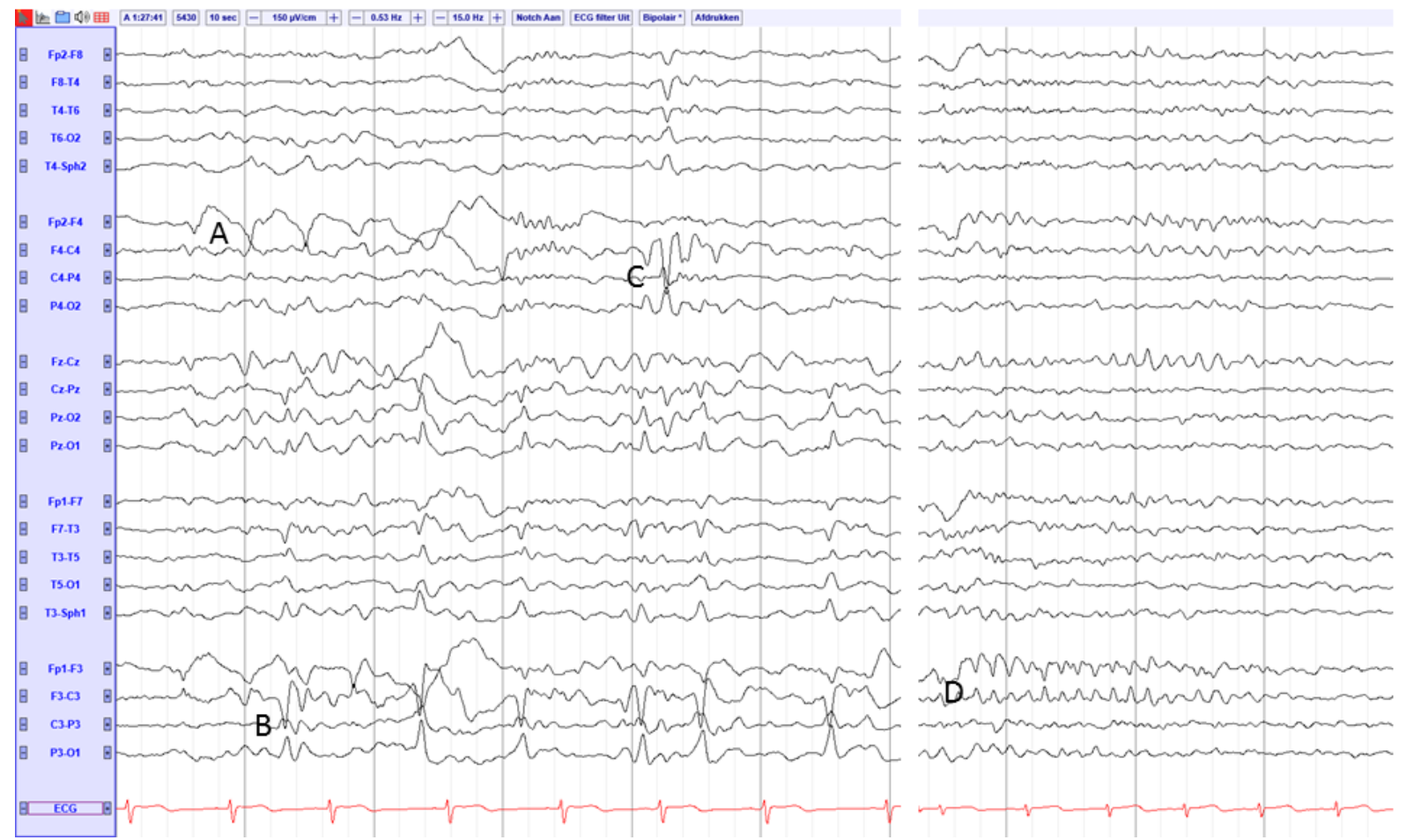

Fig. 2. EEG of individual V.6. The interictal EEG showed frequent multifocal independent spike discharges (MISD): A. right frontal (F4), B. left centroparietal (C3, P3), C. right central (C4) and left frontal (F3). Longitudinal bipolar montage. Time base: $30 \mathrm{~mm} / \mathrm{s}$; sensitivity: $150 \mu \mathrm{V} / \mathrm{cm}$; high cut: $15.0 \mathrm{~Hz}$; low cut: $0.53 \mathrm{~Hz}$.

\section{Exome sequencing}

DNA samples of the parents, one affected (V.1) and one unaffected (V.5) sibling were fragmented by sonication and libraries were prepared with the TruSeq DNA library kit (Illumina) as per the manufacturer's protocol. Exome capturing was performed using the in-solution NimbleGen SeqCap EZ Human Exome Library v2. Exome sequencing was performed on the Illumina HiSeq2000 platform. Paired-end sequencing (2X75 bp) led to an average of 5-6 Gb of data per exome. The mean coverage was 96x (Table 1).

Base-calling, alignment and variant calling were performed using the GATK package (McKenna et al., 2010). For annotation purposes, Annovar, as well as an in-house designed software Annotate-it were used (Wang et al., 2010, Sifrim et al., 2012). Data analysis was based on a recessive inheritance pattern. Although homozygous mutations seemed more likely based on the consanguinity factor, compound heterozygous variants were also taken into account. Data from linkage analysis were used as an additional filtering tool. All filtering steps included exonic nonsynonymous and splice-site variants only and presence in the 1000 Genomes Project with a maximum minor allele frequency (MAF) of 3\% (last accessed on 21/08/2014). 


\section{Sanger sequencing and X-inactivation studies}

Sanger sequencing was applied to confirm the presence of single-nucleotide variants found by exome sequencing, as described previously (Isrie et al., 2013). X-inactivation studies were also performed as described previously (Froyen et al., 2007).

\section{Expression analysis}

Samples from the proband (V.1), his father (IV.1) and four random control samples were analysed for expression levels of STYXL1 mRNA. Total RNA was extracted from EBV transformed lymphoblastoid cells using the RNeasy Mini Kit (Qiagen). cDNA synthesis was performed by taking $1 \mu \mathrm{g}$ of RNA using Superscript III FirstStrand Synthesis SuperMix for qRT-PCR (Invitrogen). Quantitative RT-PCR was performed using gene specific primers and the SYBR Green PCR Master Mix (Life Technologies) (see supplementary Table S1 for primer sequences). Relative gene expression was calculated in Microsoft Excel using the 2(- $\Delta \Delta \mathrm{Ct})$ method comparing samples and expression levels to four control samples and housekeeping genes GAPDH and GUSB. The unpaired t-test was applied for statistical purposes.

Table 1

Quality metrics of exome sequencing data (obtained from Picard metrics).

\begin{tabular}{|lcccc|}
\hline Individual & Father & Mother & Affected & Unaffected \\
\hline $\begin{array}{l}\text { Total reads } \\
\text { (number) }\end{array}$ & $127,435,484$ & $101,786,244$ & $122,610,966$ & $109,049,332$ \\
\hline $\begin{array}{l}\text { Aligned reads } \\
\text { passed filtering (\%) }\end{array}$ & 93.9 & 93.9 & 93.9 & 94.0 \\
\hline $\begin{array}{l}\text { Mean target } \\
\text { coverage (x) }\end{array}$ & 110 & 80 & 100 & 96 \\
\hline $\begin{array}{l}\text { Target bases with } \\
\geq 20 x \text { coverage (\%) }\end{array}$ & 85.2 & 80.8 & 84.0 & 83.1 \\
\hline
\end{tabular}

\section{Results}

Considering pedigree-derived information, linkage analysis and X-inactivation studies were performed. Subsequently, exome sequencing was used to seek the pathogenic mutation in this family. An overview of quality metrics of the exome sequencing is depicted in Table 1. Filtering was based on the presence of homozygous and compound heterozygous variants, respectively. In addition, a filtering approach for X-linked variants was applied, which however did not lead to any candidate variants. Eventually, qRT-PCR was performed to investigate expression levels of the most promising candidate gene. 


\section{Linkage analysis and X-inactivation studies}

Parametric linkage analysis for a recessive disorder revealed a 4.5 $\mathrm{Mb}$ region on chromosome 7 and an $11.8 \mathrm{Mb}$ region on chromosome 17, respectively. The maximum LOD-score obtained was 1.579. Furthermore, affected siblings were sharing the same haplotype block.

X-inactivation studies in the mother showed a random pattern (67/33).

\section{Filtering for homozygous variants}

Exome sequencing resulted in a total number of 36,091 variants. Table 2 illustrates the filtering strategy used, resulting in a total of eleven potentially interesting homozygous variants; of which three mapped to the respective linkage regions (see Table S2 for more details). Seven variants outside the linkage regions were validated with Sanger, however, did not segregate with the disease in the family. One other variant (SPATA31C1) was discarded because of homozygous presence in normal individuals (NHLBI Exome Variant Server - accessed 21/08/2014). The three remaining variants were missense variants in STYXL1 (p.P311A) and LRRC37A2 (p.E1224G) and a splice-site variant in MDH2 (p.K185=). Since the LRRC37A2 variant could not be validated, segregation studies of the two remaining variants were performed, confirming segregation with disease for both of them. The STYXL1 variant was predicted to be probably damaging by PolyPhen-2, SIFT as well as MutationTaster2 (Ng and Henikoff, 2003, Adzhubei et al., 2010, Schwarz et al., 2014). The MDH2 variant was predicted to be tolerated by SIFT, but damaging by MutationTaster. Since this concerned a synonymous variant, prediction through PolyPhen-2 was unavailable.

A second filtering approach focused on all homozygous variants in the linkage regions. This resulted in 584 variants. Out of these, the rare (i.e. with $1000 \mathrm{G}$ MAF $\leq 3 \%$ ) and exonic nonsynonymous or splice-site variants were retained, resulting in the same three variants as described before and thus confirming results from the previous filtering strategy.

\section{Filtering for compound heterozygous variants}

The autosomal recessive hypothesis also prompted a search for compound heterozygous mutations. A similar filtering strategy as described in Table 2 was applied to the exome sequencing data, leading to five rare compound heterozygous variants in the proband, not shared with his unaffected sibling V.5 (see Table S2). The only and most interesting candidates were two missense variants in ANK3 (p.P1489S and p.S2044G), a gene recently implicated in moderate ID, hypotonia, spasticity and behavioural problems in another consanguineous family (Iqbal et al., 2013). However, further segregation analysis indicated the presence of these compound heterozygous variants in an unaffected sibling. 
Table 2

Filtering strategy for homozygous variants.

\begin{tabular}{|l|l|}
\hline Filtering criteria & Number of variants \\
\hline total & 36,091 \\
\hline $\begin{array}{l}\text { exonic and nonsynonymous, splice-site variants or } \\
\text { indels causing frameshift }\end{array}$ & 19,287 \\
\hline homozygous in affected proband V.1 & 3,151 \\
\hline $\begin{array}{l}\text { heterozygous in parents (IV.1 and IV.2) and not } \\
\text { homozygous in unaffected sibling V.5 }\end{array}$ & 325 \\
\hline 1000G $\leq 0.03$ & 11 \\
\hline in linkage regions & 3 \\
\hline
\end{tabular}

\section{Functional investigation of candidate genes}

To our knowledge, both candidate genes, $M D H 2$ and $S T Y X L 1$, have not been implicated in a developmental phenotype before.

MDH2 (OMIM *154100) encodes a malate dehydrogenase that is bound to mitochondria in both placenta and leucocytes. Since less is known about the function of the protein and it was expected to have metabolic effects, a specific metabolic testing was performed comprising levels of organic acids (urine) and lactate (blood and urine). Both these parameters were normal in the proband and his two affected siblings. Therefore, the variant in $\mathrm{MDH} 2$ was not believed to have any pathogenic effects explaining the phenotype.

For the second candidate gene STYXL1, qRT-PCR was chosen to investigate expression levels in mRNA. Expression analysis using two probes in STYXL1 in the proband V.1 versus control samples showed a significant reduction of expression in proband V.1 ( $<$ < 0.0001) (Fig. 3). Comparison of expression levels between controls and the carrier father didn't show significant differences ( $p=0.29$ for probe 1 and $p=0.81$ for probe 2 ).

\section{Discussion}

In this study, we describe for the first time a homozygous missense mutation (p.P311A) in STYXL1, segregating with ID, seizures, and a behavioural phenotype in three siblings from a consanguineous family. The mutation was discovered by exome sequencing and linkage studies and further confirmed with segregation analysis. On mRNA level, a significant reduction in transcript levels of STYXL1 was discovered in the proband carrying a homozygous mutation and not in his carrier father. 


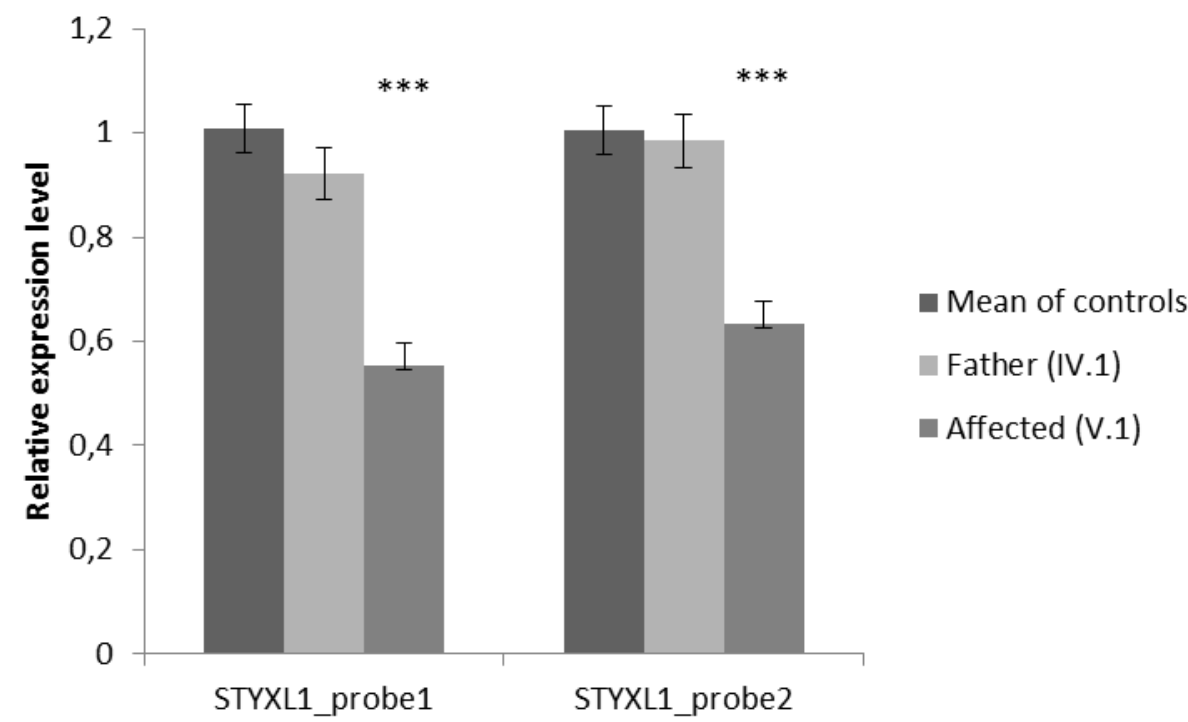

Fig. 3. Expression levels of STYXL1 mRNA in the proband and father versus controls.

STYXL1 (also known as DUSP24; MK-STYX) is officially termed serine/threonine/tyrosine interacting-like 1 gene and encodes a largely uncharacterized pseudophosphatase. It belongs to the superfamily of protein tyrosine phosphatases (PTPs), which form critical components of multiple signalling networks and are important regulators of many fundamental physiological processes. The subgroup of mitogen-activated protein kinase phosphatases (MKPs) are specifically known to dephosphorylate mitogen-activated protein kinases (MAPKs) (Patterson et al., 2009). Although part of this subgroup, STYXL1 lacks phosphatase activity due to presence of a serine residue instead of a conserved cysteine residue in the catalytic loop. Nevertheless, owing to the fact that STYX domains are evolutionarily conserved, they are expected to perform distinct non-phosphatase functions (Wishart and Dixon, 1998). One such function could for example be related to the substrate-binding activity of the STYXL1 protein that is still intact (Niemi et al., 2014).

Niemi and colleagues recently demonstrated that small interfering RNA (siRNA)-mediated knockdown of STYXL1 induces chemoresistance, while overexpression of the protein induces cell death (Niemi et al., 2011). They also showed that cell proliferation and MAPK signalling are not affected by the knockdown. Two interaction partners of STYXL1 have been described so far; PTPMT1 and G3BP1 (Hinton et al., 2010, Niemi et al., 2014). Barr and colleagues further confirmed the role of STYXL1 as a regulator in the stress response pathway (Barr et al., 2013). It can be concluded that, although a significant amount of fundamental research on STYXL1 has been conducted; little to no information is available about its role in developmental processes.

STYXL1 localizes to mitochondria (Niemi et al., 2011) and is expressed in multiple mammalian tissues. In brain, expression is high in the hippocampus, hypothalamus and the olfactory bulb (Hawrylycz et al., 2012). The gene and the mutated amino acid both are conserved among different species (Pollard et al., 2010). The deleterious effect of the p.P311A mutation was predicted by different algorithms (Ng and Henikoff, 2003, Adzhubei et al., 2010, Schwarz et al., 2014). In addition, the low frequency of heterozygous carriers, also argues for its deleterious effect (Kryukov et al., 2007). However, since the mutation is located at the end of the protein and outside a specific protein domain, it was surprising to find diminished mRNA expression levels in the 
proband. We have no readily explanation for this observation.It has been shown that codon usage influences

\section{Acknowledgements}

This work was supported by a grant from Concerted Research Actions KULeuven GOA/12/015 and funding from the Belgian Science Policy Office Interuniversity Attraction Poles (BELSPO-IAP) program through the project IAP P7/43-BeMGI. HVE is a clinical investigator of FWO-Vlaanderen. Additional funding was provided by Stichting Studiefonds KETEL 1 (2012).

\section{Supplementary data}

Table S1. Primer sequences used to detect STYXL1 mRNA expression levels with qRT-PCR

Table S2. Exome sequencing post-filtering variants, homozygous or compound heterozygous in proband V.1 


\section{References}

Abecasis GR, Cherny SS, Cookson WO, Cardon LR (2002) Merlin--rapid analysis of dense genetic maps using sparse gene flow trees. Nat Genet 30:97-101.

Adzhubei IA, Schmidt S, Peshkin L, Ramensky VE, Gerasimova A, Bork P, Kondrashov AS, Sunyaev SR (2010) A method and server for predicting damaging missense mutations. Nat Methods 7:248-249.

Barcia G, Fleming MR, Deligniere A, Gazula VR, Brown MR, Langouet M, Chen H, Kronengold J, Abhyankar A, Cilio R, Nitschke P, Kaminska A, Boddaert N, Casanova JL, Desguerre I, Munnich A, Dulac O, Kaczmarek LK, Colleaux L, Nabbout R (2012) De novo gain-of-function KCNT1 channel mutations cause malignant migrating partial seizures of infancy. Nat Genet 44:1255-1259.

Barr JE, Munyikwa MR, Frazier EA, Hinton SD (2013) The pseudophosphatase MK-STYX inhibits stress granule assembly independently of Ser149 phosphorylation of G3BP-1. Febs J 280:273-284.

de Ligt J, Willemsen MH, van Bon BW, Kleefstra T, Yntema HG, Kroes T, Vulto-van Silfhout AT, Koolen DA, de Vries P, Gilissen C, del Rosario M, Hoischen A, Scheffer H, de Vries BB, Brunner HG, Veltman JA, Vissers LE (2012) Diagnostic exome sequencing in persons with severe intellectual disability. N Engl J Med 367:1921-1929.

Froyen G, Van Esch H, Bauters M, Hollanders K, Frints SG, Vermeesch JR, Devriendt K, Fryns JP, Marynen P (2007) Detection of genomic copy number changes in patients with idiopathic mental retardation by high-resolution X-array-CGH: important role for increased gene dosage of XLMR genes. Hum Mutat 28:1034-1042.

Guigo R (1999) DNA composition, codon usage and exon prediction. In: Genetic databases (M, B., ed), pp 53-80: Academic press.

Hawrylycz MJ, Lein ES, Guillozet-Bongaarts AL, Shen EH, Ng L, Miller JA, van de Lagemaat LN, Smith KA, Ebbert A, Riley ZL, Abajian C, Beckmann CF, Bernard A, Bertagnolli D, Boe AF, Cartagena PM, Chakravarty MM, Chapin M, Chong J, Dalley RA, Daly BD, Dang C, Datta S, Dee N, Dolbeare TA, Faber V, Feng D, Fowler DR, Goldy J, Gregor BW, Haradon Z, Haynor DR, Hohmann JG, Horvath S, Howard RE, Jeromin A, Jochim JM, Kinnunen M, Lau C, Lazarz ET, Lee C, Lemon TA, Li L, Li Y, Morris JA, Overly CC, Parker PD, Parry SE, Reding M, Royall JJ, Schulkin J, Sequeira PA, Slaughterbeck CR, Smith SC, Sodt AJ, Sunkin SM, Swanson BE, Vawter MP, Williams D, Wohnoutka P, Zielke HR, Geschwind DH, Hof PR, Smith SM, Koch 
C, Grant SG, Jones AR (2012) An anatomically comprehensive atlas of the adult human brain

transcriptome. Nature 489:391-399.

Heron SE, Grinton BE, Kivity S, Afawi Z, Zuberi SM, Hughes JN, Pridmore C, Hodgson BL, Iona X, Sadleir LG, Pelekanos J, Herlenius E, Goldberg-Stern H, Bassan H, Haan E, Korczyn AD, Gardner AE, Corbett MA, Gecz J, Thomas PQ, Mulley JC, Berkovic SF, Scheffer IE, Dibbens LM (2012a) PRRT2 mutations cause benign familial infantile epilepsy and infantile convulsions with choreoathetosis syndrome. Am J Hum Genet 90:152-160.

Heron SE, Smith KR, Bahlo M, Nobili L, Kahana E, Licchetta L, Oliver KL, Mazarib A, Afawi Z, Korczyn A, Plazzi G, Petrou S, Berkovic SF, Scheffer IE, Dibbens LM (2012b) Missense mutations in the sodium-gated potassium channel gene KCNT1 cause severe autosomal dominant nocturnal frontal lobe epilepsy. Nat Genet 44:1188-1190.

Hinton SD, Myers MP, Roggero VR, Allison LA, Tonks NK (2010) The pseudophosphatase MK-STYX interacts with G3BP and decreases stress granule formation. Biochem J 427:349-357.

Iqbal Z, Vandeweyer G, van der Voet M, Waryah AM, Zahoor MY, Besseling JA, Roca LT, Vulto-van Silfhout AT, Nijhof B, Kramer JM, Van der Aa N, Ansar M, Peeters H, Helsmoortel C, Gilissen C, Vissers LE, Veltman JA, de Brouwer AP, Frank Kooy R, Riazuddin S, Schenck A, van Bokhoven H, Rooms L (2013) Homozygous and heterozygous disruptions of ANK3: at the crossroads of neurodevelopmental and psychiatric disorders. Hum Mol Genet 22:1960-1970.

Isrie M, Kalscheuer VM, Holvoet M, Fieremans N, Van Esch H, Devriendt K (2013) HUWE1 mutation explains phenotypic severity in a case of familial idiopathic intellectual disability. Eur J Med Genet 56:379-382.

Kong A, Gudbjartsson DF, Sainz J, Jonsdottir GM, Gudjonsson SA, Richardsson B, Sigurdardottir S, Barnard J, Hallbeck B, Masson G, Shlien A, Palsson ST, Frigge ML, Thorgeirsson TE, Gulcher JR, Stefansson K (2002) A high-resolution recombination map of the human genome. Nat Genet 31:241-247.

Kryukov GV, Pennacchio LA, Sunyaev SR (2007) Most rare missense alleles are deleterious in humans: implications for complex disease and association studies. Am J Hum Genet 80:727-739.

Krzywinski M, Schein J, Birol I, Connors J, Gascoyne R, Horsman D, Jones SJ, Marra MA (2009) Circos: an information aesthetic for comparative genomics. Genome Res 19:1639-1645. 
McKenna A, Hanna M, Banks E, Sivachenko A, Cibulskis K, Kernytsky A, Garimella K, Altshuler D, Gabriel S, Daly M, DePristo MA (2010) The Genome Analysis Toolkit: a MapReduce framework for analyzing nextgeneration DNA sequencing data. Genome Res 20:1297-1303.

Najmabadi H, Hu H, Garshasbi M, Zemojtel T, Abedini SS, Chen W, Hosseini M, Behjati F, Haas S, Jamali P, Zecha A, Mohseni M, Puttmann L, Vahid LN, Jensen C, Moheb LA, Bienek M, Larti F, Mueller I, Weissmann R, Darvish H, Wrogemann K, Hadavi V, Lipkowitz B, Esmaeeli-Nieh S, Wieczorek D, Kariminejad R, Firouzabadi SG, Cohen M, Fattahi Z, Rost I, Mojahedi F, Hertzberg C, Dehghan A, Rajab A, Banavandi MJ, Hoffer J, Falah M, Musante L, Kalscheuer V, Ullmann R, Kuss AW, Tzschach A, Kahrizi K, Ropers HH (2011) Deep sequencing reveals 50 novel genes for recessive cognitive disorders. Nature 478:57-63.

Ng PC, Henikoff S (2003) SIFT: Predicting amino acid changes that affect protein function. Nucleic Acids Res 31:3812-3814.

Niemi NM, Lanning NJ, Klomp JA, Tait SW, Xu Y, Dykema KJ, Murphy LO, Gaither LA, Xu HE, Furge KA, Green DR, MacKeigan JP (2011) MK-STYX, a catalytically inactive phosphatase regulating mitochondrially dependent apoptosis. Mol Cell Biol 31:1357-1368.

Niemi NM, Sacoman JL, Westrate LM, Gaither LA, Lanning NJ, Martin KR, Mackeigan JP (2014) The Pseudophosphatase MK-STYX Physically and Genetically Interacts with the Mitochondrial Phosphatase PTPMT1. PLoS One 9:e93896.

Novoa EM, Ribas de Pouplana L (2012) Speeding with control: codon usage, tRNAs, and ribosomes. Trends Genet 28:574-581.

Patterson KI, Brummer T, O'Brien PM, Daly RJ (2009) Dual-specificity phosphatases: critical regulators with diverse cellular targets. Biochem J 418:475-489.

Pollard KS, Hubisz MJ, Rosenbloom KR, Siepel A (2010) Detection of nonneutral substitution rates on mammalian phylogenies. Genome Res 20:110-121.

Rauch A, Wieczorek D, Graf E, Wieland T, Endele S, Schwarzmayr T, Albrecht B, Bartholdi D, Beygo J, Di Donato N, Dufke A, Cremer K, Hempel M, Horn D, Hoyer J, Joset P, Ropke A, Moog U, Riess A, Thiel CT, Tzschach A, Wiesener A, Wohlleber E, Zweier C, Ekici AB, Zink AM, Rump A, Meisinger C, Grallert H, Sticht H, Schenck A, Engels H, Rappold G, Schrock E, Wieacker P, Riess O, Meitinger T, Reis A, Strom TM (2012) Range of genetic mutations associated with severe non-syndromic sporadic intellectual disability: an exome sequencing study. Lancet 380:1674-1682. 
Schwarz JM, Cooper DN, Schuelke M, Seelow D (2014) MutationTaster2: mutation prediction for the deepsequencing age. Nat Methods 11:361-362.

Sifrim A, Van Houdt JK, Tranchevent LC, Nowakowska B, Sakai R, Pavlopoulos GA, Devriendt K, Vermeesch JR, Moreau Y, Aerts J (2012) Annotate-it: a Swiss-knife approach to annotation, analysis and interpretation of single nucleotide variation in human disease. Genome Med 4:73.

Wang K, Li M, Hadley D, Liu R, Glessner J, Grant SF, Hakonarson H, Bucan M (2007) PennCNV: an integrated hidden Markov model designed for high-resolution copy number variation detection in whole-genome SNP genotyping data. Genome research 17:1665-1674.

Wang K, Li M, Hakonarson H (2010) ANNOVAR: functional annotation of genetic variants from high-throughput sequencing data. Nucleic Acids Res 38:e164.

Wishart MJ, Dixon JE (1998) Gathering STYX: phosphatase-like form predicts functions for unique proteininteraction domains. Trends Biochem Sci 23:301-306.

Xiong HY, Alipanahi B, Lee LJ, Bretschneider H, Merico D, Yuen RKC, Hua Y, Gueroussov S, Najafabadi HS, Hughes TR, Morris Q, Barash Y, Krainer AR, Jojic N, Scherer SW, Blencowe BJ, Frey BJ (2015) The human splicing code reveals new insights into genetic determinants of disease. Science 347

\title{
Web Resources \& URLs
}

\section{Genomes}

http://browser.1000genomes.org/index.html

\section{Allen Institute for Brain Science, Allen Human Brain Atlas}

http://human.brain-map.org/

\author{
Annotate-it \\ http://www.annotate-it.org/ \\ Circos (data visualization) \\ http://circos.ca/
}

Database of Single Nucleotide Polymorphisms (dbSNP). Bethesda (MD): National Center for Biotechnology Information, National Library of Medicine 
http://www.ncbi.nlm.nih.gov/SNP/

1

2

Exome Variant Server, NHLBI GO Exome sequencing project (ESP), Seattle, WA

http://evs.gs.washington.edu/EVS/ [June 2014]

\section{MutationTaster}

http://mutationtaster.org/

\section{Picard metrics}

http://picard.sourceforge.net/index.shtml/

\section{PolyPhen-2}

http://genetics.bwh.harvard.edu/pph2/

$\mathbf{R}$ (data visualization)

http://cran.r-project.org

\section{SUPPLEMENTARY DATA}

Table S1

Primers used for quantitative RT-PCR

\begin{tabular}{|l|l|l|}
\hline STYXL1_ex3_FWD & GTGATCACTGCCCTTCGAGT & \\
\hline STYXL1_ex4_REV & CACACACTCCAGGTCCACAG & $75 \mathrm{bp}$ \\
\hline STYXL1_ex8_FWD & CCGCCATCATAGCCTACCT & \\
\hline STYXL1_ex9_REV & CAATCCCCGATTTGGACA & $104 \mathrm{bp}$ \\
\hline
\end{tabular}

Table S2

Exome sequencing post-filtering variants, homozygous or compound heterozygous in proband V.1. (MAF=minor allele frequency; $\mathrm{n} / \mathrm{a}=$ not applicable) 
To Professor Dr. A. Verloes

Leuven, 14-01-2015

Editor

European Journal of Medical Genetics

Dept. of Medical Genetics, Clinical Genetic Unit

CHU Robert Debré

48 Boulevard Sérurier

75935 Paris

France

Dear Professor Verloes,

Thank you very much for the review of our Exome Report entitled "Homozygous missense mutation in STYXL1 associated with moderate intellectual disability, epilepsy and behavioural complexities", manuscript n ${ }^{\circ}$ EJMG-D-14-00412.

We appreciate the time and useful comments of both reviewers, and would like to submit a revised version of our manuscript. In this revised manuscript we have attended to all the comments and points raised by the reviewers, and these are detailed below.

\section{Reviewer \#1}

1. From analyzing the EXAC exome variant database, it seems the STYXL1 variant is not so rare in the African populations, with $4 \%$ being carriers and 3 homozygous individuals in the database. It is difficult to reconcile this with the stated hypothesis that this variant is a cause for ID and epilepsy.

R: This new exome database was launched recently, after submission of our manuscript and indeed this is a very useful database. However, we believe that one needs to interpret the data with caution since the exomes and genotype data present in this database are both from healthy and disease-specific populations, including several psychiatric and behavioral disorders. We have no further data on the 3 homozygous individuals, to which study they belonged and how well they were phenotyped. Especially, given the mild and psychiatric phenotypes in our individuals, this might be overlooked.

Moreover, the goal of the "Exome Report" section, if we understand well, is the dissemination of possible genotype-phenotype correlations without the necessity to reach strong functional evidences, or to wait a second case for data replication. The variants are predicted to be pathogenic by all prediction programs used, they segregate in the family and match the homozygousity data.

2. It is not clear to me why the authors decided to look at mRNA expression levels for a missense-encoding SNP. Also, the stated hypotheses in the discussion as to why the mRNA levels would be lower are not convincing and in one case not valid.

$\mathbf{R}$ : We agree that the changes in protein confirmation do not affect mRNA expression levels and have deleted this in the manuscript's discussion. Since we received a cell line, we checked mRNA levels and found to our surprise a much lower level in the affected versus the controls, even after repeating the experiment with different primer sets. Missense mutation usually do not trigger non-sense mediated decay, so we needed to think about other potential hypotheses. We 
have added another hypotheses, regarding splicing, recently described by Xiong HY and coworkers in Science 2015.

\section{Reviewer \#2}

1. Both the Figure 1 and 2 (except the pedigree chart) are not informative at all, and it is beyond our limit visually, to read the figures. The info in the figures can just be described in the text. Please consider to put these figures in supplementary.

R: We have uploaded the Figures separately. Figure 1 shows the results of the homozygosity mapping performed in all members of the family, and shows the linkage interval and the position of the STYXL1 gene.

2. Why homozygosity mapping approach was not considered in the affected and unaffected children, which is a powerful method to narrow down the regions for recessive causative mutations especially in the case of consanguineous.

R: Homozygosity mapping was performed in all members of the family, as stated in the submitted manuscript $\mathrm{p} 7$ and Figure 1.

3. Why the mutation in STYXL1 was not tested in other affected and unaffected children (although it has been shown indirectly through segregation)?

R: It was tested in all children, both affected and normal, and segregates with the phenotype, this was stated in the submitted manuscript $\mathrm{p} 7$.

4. Please explain why a MAF $3 \%$ was used in filtering? Any data to support this cutoff? R: To our knowledge, there's as yet no consensus on the ideal filtering approach in wholeexome sequencing for recessive disorders. In this family, we were looking for an autosomal recessive pathogenic variant leading to non-syndromic ID and epilepsy. Therefore, we could allow a certain number of carriers in the normal population. Previous studies mention cut-offs of $0.5-2 \%$ and in order to make sure not to miss any relevant variants, we decided to use the less stringent $3 \%$. The access of variants that this relatively high percentage would cause, could subsequently be filtered out by other methods, e.g. segregation in the siblings, as is described in the results section.

5. Why filtering approach for X-linked variants was applied? Are the affected children all males, or $\mathrm{x}$-linked transmission is suspected?

$\mathbf{R}$ : Because the 3 affected children are male, we did want to exclude an X-linked cause (see pedigree Figure 1). That is the reason for doing $\mathrm{X}$-inactivation studies in the mother and specifically filtering for $\mathrm{X}$-linked variants.

6. Any analysis had been done using tools such as PolyPhen, SIFT etc to predict the functional impacts and evolutionary conservation of the variants found in the 2 potential candidate genes? R: This analysis was done but we now have explicitly added the outcomes of SIFT, PolyPhen-2 and MutationTaster 2 predictions in the results section. 
We hope that we have fully addressed all comments of the reviewers to their satisfaction and that you will consider this revised manuscript for publication.

\author{
Yours sincerely, \\ Dr. Hilde Van Esch \\ Center for Human Genetics \\ Herestraat 49 \\ B-3000 Leuven \\ Belgium \\ Tel : +32-16-345903 \\ Fax : +32-16-346051 \\ E-mail : $\underline{\text { Hilde.VanEsch@med.kuleuven.ac.be }}$
}


Figure 1

Click here to download high resolution image

a

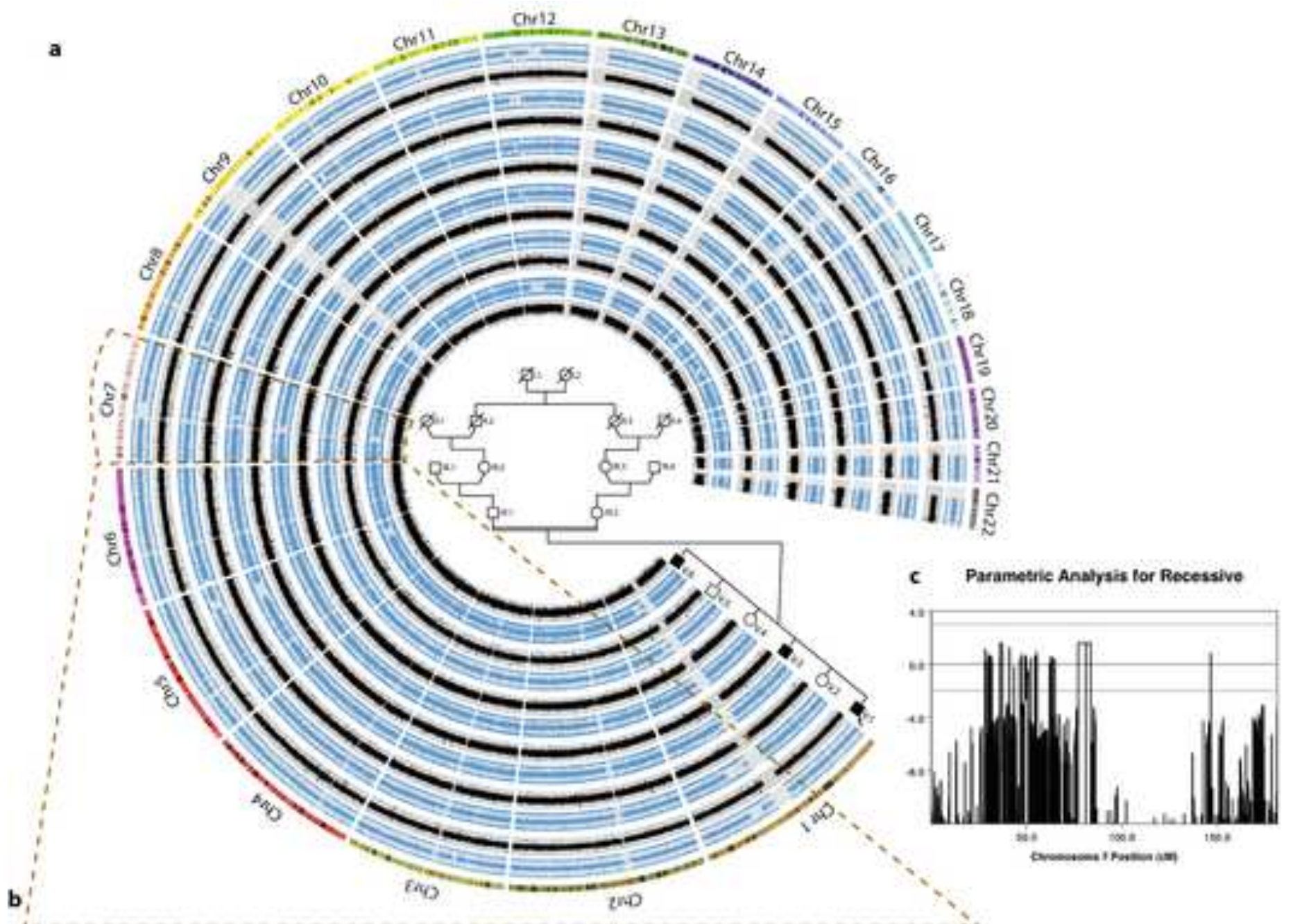

b!

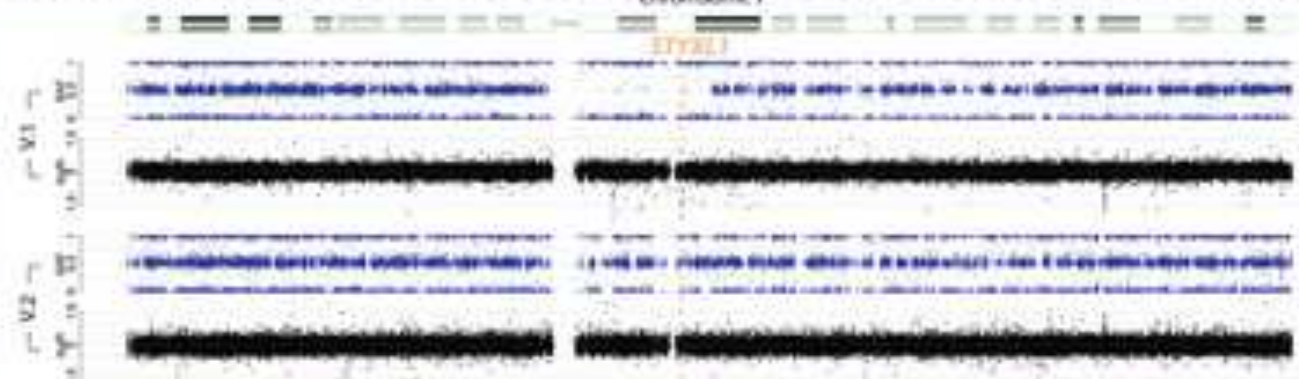

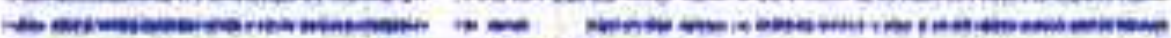

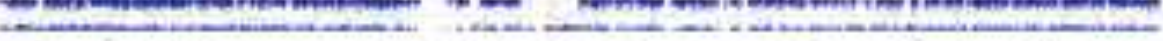
H.

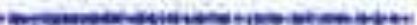

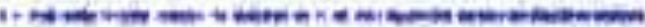

-

$\$$

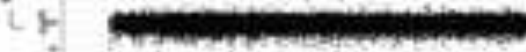

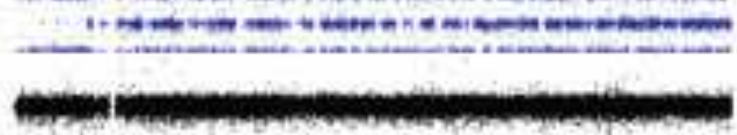

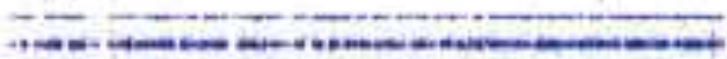

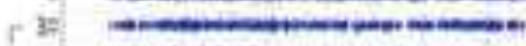

$\geqslant:$

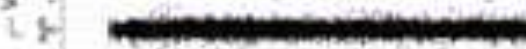

-

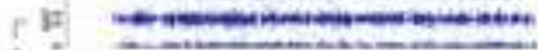

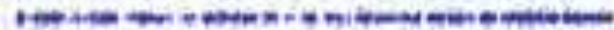

$3:$

4.

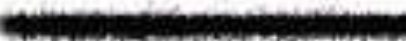

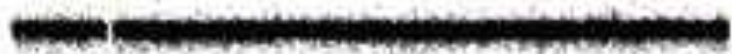

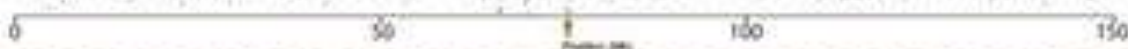


Click here to download high resolution image
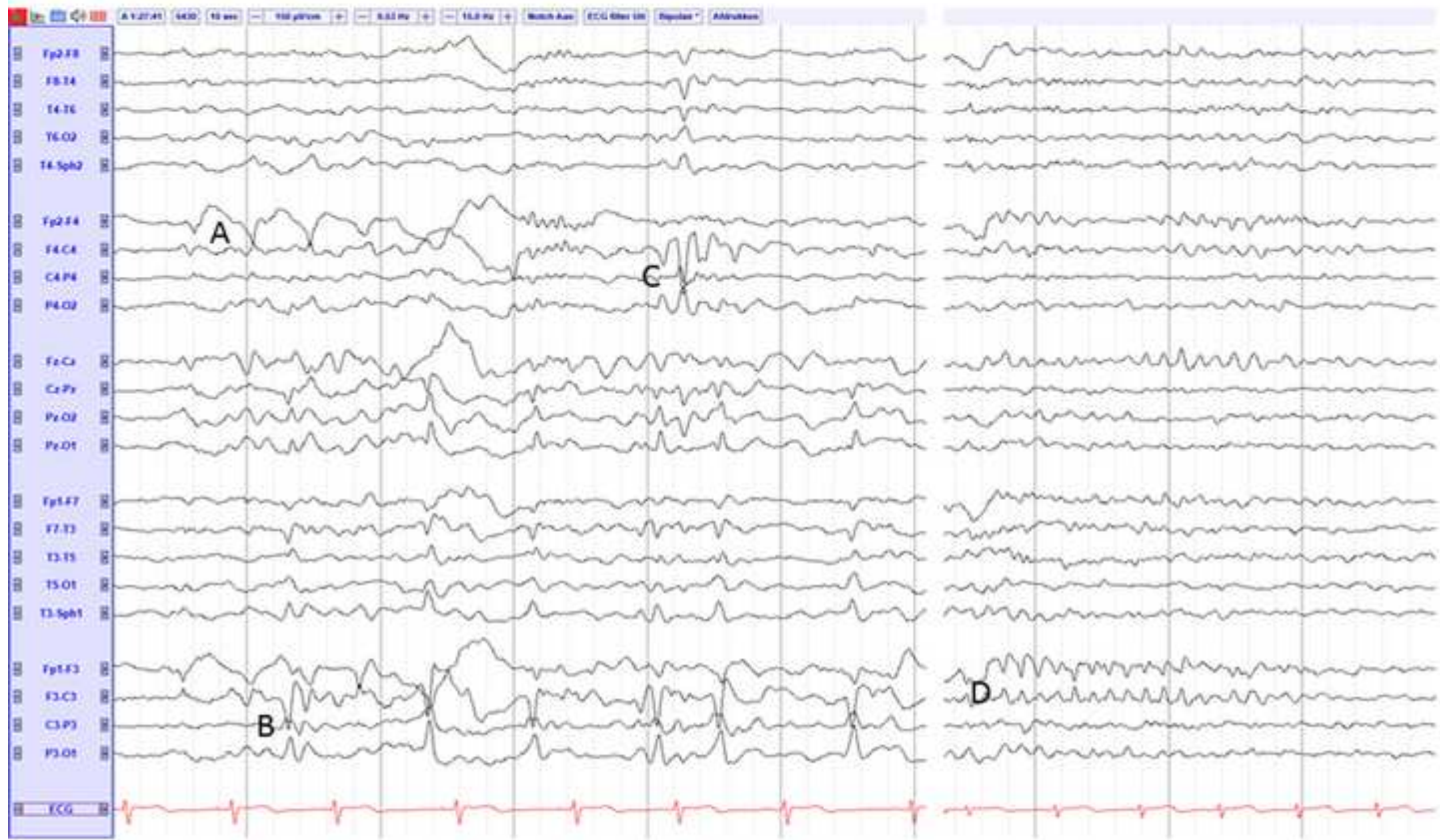


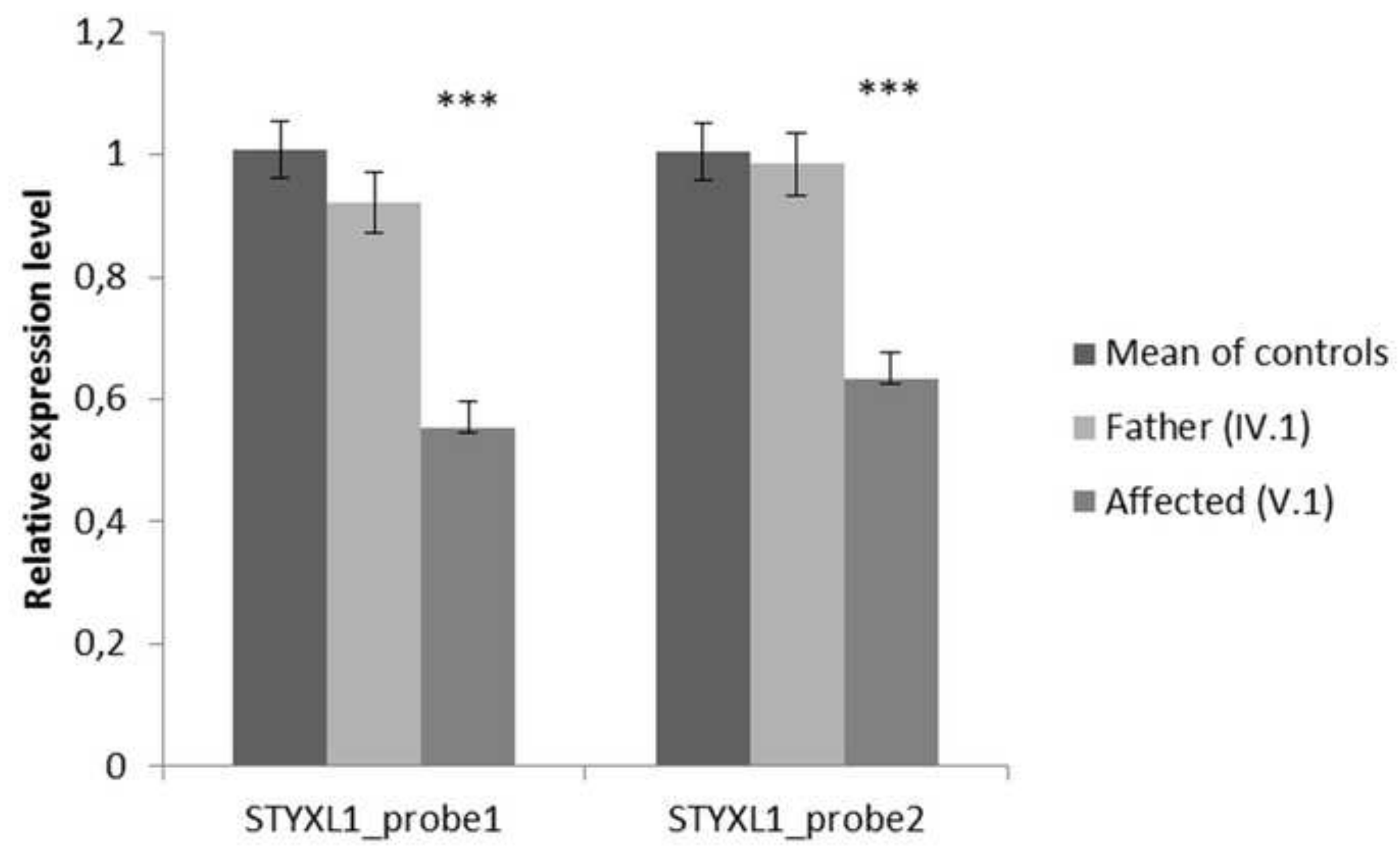

